\title{
Search for bound-state electron+positron pair decay
}

\author{
F. Bosch ${ }^{1}$, S. Hagmann ${ }^{1}$, P.-M. Hillenbrand ${ }^{1}$, G. J. Lane ${ }^{2}$, Yu. A. Litvinov ${ }^{1,3, a}$, M. W. Reed ${ }^{2, b}$, M. S. Sanjari ${ }^{1}$, \\ Th. Stöhlker ${ }^{1,4,5}$, S. Yu. Torilov ${ }^{6}$, X. L. Tu ${ }^{1,3,7}$, and P. M. Walker ${ }^{8}$ \\ ${ }^{1}$ GSI Helmholtzzentrum für Schwerionenforschung, Planckstraße 1, 64291 Darmstadt, Germany \\ ${ }^{2}$ Department of Nuclear Physics, Research School of Physics and Engineering, \\ The Australian National University, Canberra, ACT 2601, Australia \\ ${ }^{3}$ Max-Planck-Institut für Kernphysik, Saupfercheckweg 1, 69117 Heidelberg, Germany \\ ${ }^{4}$ Helmholtz-Institut Jena, 07743 Jena, Germany \\ ${ }^{5}$ Institut für Optik und Quantenelektronik, Friedrich-Schiller-Universität Jena, 07743 Jena, Germany \\ ${ }^{6}$ St. Petersburg State University, Ulyanovskaya str. 1, Petrodvorets, 198504 St. Petersburg, Russia \\ ${ }^{7}$ Institute of Modern Physics, Chinese Academy of Sciences, Lanzhou 730000, China \\ ${ }^{8}$ Department of Physics, University of Surrey, Guildford, Surrey GU2 7XH, United Kingdom
}

\begin{abstract}
. the heavy ion storage rings coupled to in-flight radioactive-ion beam facilities, namely the ability to produce and store for extended periods of time radioactive nuclides in high atomic charge states, for the search of yet unobserved decay mode - bound-state electron-positron pair decay.
\end{abstract}

\section{Introduction}

The question on whether nuclear halflives are fundamental constants or can be modified by external manipulations was asked already at the beginning of nuclear physics [1]. Only tiny effects of below about one percent were found, see review [2]. Recently, a $1.5 \%$ change in the electron capture decay rate of ${ }^{7} \mathrm{Be}$ implanted in $\mathrm{C}_{60}$ clusters was observed [3]. Unconfirmed and disputed claims exist on the dramatic acceleration of $\alpha$-decay in nuclei implanted into metals at cryogenic temperatures [4]. In all these cases, the results were explained using the modifications of electron densities, which is one of the main quantities needed to describe decay processes involving bound electrons [5].

Even with this scarce experimental evidence, it has been observed that high atomic charge states can dramatically modify nuclear decay properties [6-8]. A striking example is ${ }^{163} \mathrm{Dy}$. As a neutral atom it is stable. However, when fully-ionised its halflife becomes merely $T_{1 / 2}\left({ }^{163} \mathrm{Dy}^{64+}\right) \sim 33$ years [9]. In the case of ${ }^{187} \mathrm{Re}$, removing all orbital electrons reduces the half-life by nine orders of magnitude [10]. Both nuclides decay via boundstate $\beta$ decay in which the decay electron occupies one of the bound orbitals instead of being emitted to the continuum. This decay mode is a time-mirror of the electron capture decay. The process is marginal in neutral atoms, but it can be significant if atoms are highly-ionised.

It is immediately obvious that orbital electron capture and internal conversion decays are disabled in fullyionised atoms. In such cases weak decay branches

\footnotetext{
a e-mail: Y.Litvinov@gsi.de

be-mail: Matthew.Reed@anu.edu.au
}

like positron emission (if energetically allowed) or $\gamma d e$ excitation can be investigated. For instance, conversion coefficients of nuclear isomers [11-13], were measured in fully ionised ${ }^{144} \mathrm{~Tb},{ }^{149} \mathrm{Dy}$ and ${ }^{151} \mathrm{Er}$ isomeric states. Investigations of decay probabilities as a function of atomic charge states reveal interesting results. Counterintuitive results were obtained in electron capture of one- and twoelectron systems in ${ }^{122} \mathrm{I},{ }^{140} \mathrm{Pr}$ and ${ }^{142} \mathrm{Pm}$ ions [14-16], where the rate is by about $50 \%$ larger in the ion with one bound electron as compared to the ion with two electrons. These results can be explained by taking into consideration the conservation of the total nucleus plus leptons angular momentum $[17,18]$. Due to the energetic blocking of the $K$-shell internal conversion, the decay rate of the $35.5-\mathrm{keV}$ first excited state in ${ }_{52}^{125} \mathrm{Te}$ is increased by $300 \%$ and $640 \%$ for $47+$ and $48+$ charge states as compared to the value known for neutral atoms [19]. A new decay mode boundstate internal conversion was observed for few-electron $\mathrm{Fe}$ isomers [20, 21]. Here the isomer de-excitation energy is transferred to a bound electron which is then excited to a higher, unoccupied atomic level. Increased half-life of the isomeric state in ${ }^{192}$ Os with a single bound electron allowed for benchmarking conversion coefficient estimations [22]. Studying the decays of isomers in highlycharged ions is important not only for investigations of weak decay branches or for testing of theoretical calculations, it is essential also for nuclear astrophysics [23], where the pathways of nucleosynthesis may dramatically be altered if low-spin long-lived excited states are present [24]. In particular this relates to $0^{+} \rightarrow 0^{+}(E 0)$ transitions which are strongly suppressed in fully-ionised atoms. 


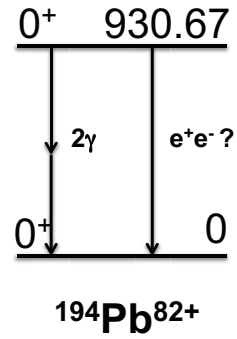

Figure 1. The decay scheme of the first excited state in ${ }^{194} \mathrm{~Pb}$.

\section{Bound-state electron-positron pair decay}

Since a photon is a boson with unit spin, the $0^{+} \rightarrow 0^{+}$ transitions via a single photon emission are forbidden. In neutral atoms such decays proceed via internal conversion or, if the transition energy is larger than two times the rest mass of electron $m_{e^{-}}=511 \mathrm{keV}$, via electron-positron pair decay, $e^{-}+e^{+}$. Furthermore, double gamma-ray emission is possible [25-28].

In the completely ionised atom, decay via internal conversion is impossible and, if $e^{-}+e^{+}$is energetically disabled, the half-life of the state can dramatically be extended since the double $\gamma$-ray decay is slow (second order in $\alpha, \propto \alpha^{2}$ ). This can have dramatic consequence, for instance, for rapid proton-capture nucleosynthesis process running along the $N=Z$ line, where many even-even nuclei with first excited $0^{+}$states are known.

In this paper we propose to search for a bound-state electron-positron pair decay, the yet unobserved decay mode. E0 decays with transition energies below the 1022$\mathrm{keV}$ pair production threshold exist in neutron-deficient lead nuclei, where low-lying $0^{+}$states are a consequence of nuclear shape coexistence [29].

One of the prominent candidates for this study is the magic ${ }_{82}^{194} \mathrm{~Pb}_{112}^{82+}$. The decay scheme of the first excited state in ${ }^{194} \mathrm{~Pb}$ is illustrated in Fig. 1 . The energy of the $0^{+}$state is $E\left(0^{+}\right)=930.67 \mathrm{keV}$ which is known from precision spectroscopy investigations [30]. This energy is below $1022-\mathrm{keV}$ threshold for $e^{-}+e^{+}$decay. However, the binding energy of the last $K$-electron is $B(K)=101.336 \mathrm{keV}$ $[31,32]$, which makes the new decay mode, bound-state $e^{-}+e^{+}$decay, energetically allowed. In this case the electron shall occupy the vacant $K$-shell thus saving $100 \mathrm{keV}$ energy. The excess energy of about $10 \mathrm{keV}$ is taken away by a monochromatic positron, which may appear as a forward-emitted particle in the laboratory frame.

\section{Experiment}

It is proposed to employ a heavy-ion storage ring coupled to an radioactive-ion beam facility for the search of the bound-state $e^{-}+e^{+}$decay. Ions of ${ }^{194} \mathrm{~Pb}$ produced in the first excited state can be stored as fully-ionised atoms in a ring. The quantum state in which ${ }^{194} \mathrm{~Pb}$ are stored will continuously be monitored by the time-resolved Schottky mass spectrometry (SMS) $[33,34]$. Nuclides with different masses are resolved by their revolution frequencies. The noise power of the Fourier-transformed signal directly relates to the number of stored ions. The SMS is sensitive to single particles [35] and has been employed for the search of long-lived isomers [36-39] as well as new isotopes $[40,41]$. With new resonant detectors, time resolution of a few milliseconds can be envisioned [42-44]. The mass resolving power of the SMS is about 700000 [45].

The decay in the ring is characterised by the disappearance of the signal at the revolution frequency of the parent ion and the correlation in time to the appearance of a signal at the frequency corresponding to the daughter ion [46]. The change in frequency reflects the change in the mass-over-charge ratio between the parent and the daughter ions. The details on the single-particle decay spectroscopy in storage rings can be found in [47].

Isomeric states decaying by double gamma-ray emission will not change the charge state and the daughter ion is still ${ }^{194} \mathrm{~Pb}^{82+}$ in its ground state. The bound-state $e^{-}+e^{+}$ decay results in ${ }^{194} \mathrm{~Pb}^{81+}$, which has a distinctly different revolution frequency. There is however a source of background, which is due to atomic pick-up of an electron from the rest-gas yielding the same daughter ions. If electron cooling of the stored ions [48] is employed, then also the recombination with the cooler electron has to be considered. Alternatively to electron cooling, the ions can be cooled stochastically $[49,50]$ or the ring can be operated in the isochronous ion-optical mode $[45,51,52]$.

To perform first feasibility studies, one could use the operating facility at GSI Helmholtzentrum for Heavy Ion Research in Darmstadt, Germany, which is a combination of the fragment separator FRS [53] and the experimental storage ring ESR [54]. One could, for instance, study the most probable way to produce the first $0^{+}$in ${ }^{194} \mathrm{~Pb}^{82+}$. Direct production at the FRS or the production directly in the ESR shall be considered. In-ring $\alpha$-decays were suggested for the ESR to study the electron screening effects [55], though no systematic investigations were performed so far (see [56] for more details). The $\alpha$-decay branchings ${ }_{84}^{198} \mathrm{Po}$ populating the states in ${ }^{194} \mathrm{~Pb}^{82+}$ are known [30], and the in-ring $\alpha$-decay decay might be an efficient method to produce the state of interest. Concerning the background, one way to remove it could be the extraction and storage of ions in a Penning trap coupled to the ESR, HITRAP [57]. In this case, a clean detection method would be to measure $11-\mathrm{keV}$ positrons from the decay. A possibility to distinguish the atomic electron capture from the isomer de-excitation decays on the basis of the distributions of the ion recoils (see [47]) shall be investigated as well. Monochromatic positrons emitted by fast projectiles is a favourable background-free detection method. Detection of $\sim 11-\mathrm{keV}$ positrons is feasible with an electronspectrometer installed at the ESR [58,59]. The concept of detecting positrons is described in detail in [60].

With higher kinetic energies the probability to pick-up electrons rapidly decreases. Therefore, another facility for the experiment could be the Facility for Antiproton and Ion Research, FAIR, which is under construction in Darm- 
stadt [61]. There, the new fragment separator Super-FRS [62] and the isochronous Collector Ring [63] would allow for such kind of experiment at a much higher energy (740 $\mathrm{MeV} / \mathrm{u})$ than presently possible at GSI $(400 \mathrm{MeV} / \mathrm{u})$. We note that the High Energy Storage Ring, HESR, will allow for storing ion beams to energies of up to $5 \mathrm{GeV} / \mathrm{u}$ $[64,65]$. However, the acceleration time of several ten seconds is not suitable for the present experiment. Similar to FAIR, such experiment could be considered for the High Intensity Accelerator Facility, HIAF, which is in a planning stage in China [66].

The highest primary beam intensities are presently available at RIKEN Nishina Center in Wako, Japan. Here an isochronous storage ring Rare RI Ring (R3) [67, 68] coupled to the BigRips fragment separator was commissioned in 2015. Due to the DC-nature of the RIKEN main cyclotron, individual ions are identified in flight and are injected into the R3 at about $400 \mathrm{MeV} / \mathrm{u}$. This energy is the same as can be achieved at GSI. However, dependent on the production rate of the $0^{+}$state of interest, the facility in RIKEN can be superior for this experiment.

\section{Summary}

The bound-state $e^{-}+e^{+}$decay is energetically allowed for the first $0^{+}$excited state in fully-ionised ${ }^{194} \mathrm{~Pb}^{82+}$. The observation of this decay mode can experimentally be addressed at heavy-ion storage rings coupled to the radioactive-ion production facilities [69]. Such experiments, however, are very difficult at present but can be planned at the new generation radioactive-ion beam facilities like FAIR or HIAF.

\section{In memorium}

This short paper is dedicated to George Dracoulis, our friend who was inspired by the application of the heavyion storage rings to isomer studies.

\section{Acknowledgements}

This work is supported in part by the Helmholtz-CAS Joint Research Group (HCJRG-108), the Australian Research Council (DP120101417 and FT100100991), and the UKScience and Technology Facilities Council.

\section{References}

[1] E. Rutherford and F. Soddy, J. Chem. Soc., Trans. 81, 837 (1902).

[2] G. T. Emery, Ann. Rev. Nucl. Sci. 22, 165 (1972).

[3] T. Ohtsuki, K. Ohno, T. Morisato et al., Phys. Rev. Lett. 98, 252501 (2007).

[4] F. Raiola, T. Spillane, B. Limata et al., Eur. Phys. J. A 32, 51 (2007).

[5] W. Bambynek, H. Behrens, M. H. Chen et al., Rev. Mod. Phys. 49, 77 (1977).
[6] Y. A. Litvinov and F. Bosch, Rep. Prog. Phys. 74, 016301 (2011).

[7] F. Bosch, Y. A. Litvinov and T. Stöhlker, Prog. Part. Nucl. Phys. 73, 84 (2013).

[8] D. Atanasov, K. Blaum, F. Bosch et al., J. Phys. B: At. Mol. Opt. 48, 144024 (2015).

[9] M. Jung, F. Bosch, K. Beckert et al., Phys. Rev. Lett. 69, 2164 (1992).

[10] F. Bosch, T. Faestermann, J. Friese et al., Phys. Rev. Lett. 77, 5190 (1996).

[11] P. Walker and G. Dracoulis, Nature 399, 35 (1999).

[12] P. M. Walker and G. D. Dracoulis, Hyperfine Interact. 135, 83 (2001).

[13] G. D. Dracoulis, Phys. Scripta T152, 014015 (2013).

[14] Y. A. Litvinov, F. Bosch, H. Geissel et al., Phys. Rev. Lett. 99, 262501 (2007).

[15] N. Winckler, H. Geissel, Y. Litvinov et al., Phys. Lett. B 679, 36 (2009).

[16] D. R. Atanasov, N. Winckler, D. Balabanski et al., Eur. Phys. J. A 48, 22 (2012).

[17] Z. Patyk, J. Kurcewicz, F. Bosch et al., Phys. Rev. C 77, 014306 (2008).

[18] K. Siegień-Iwaniuk, N. Winckler, F. Bosch et al., Phys. Rev. C 84, 014301 (2011).

[19] F. Attallah, M. Aiche, J. F. Chemin et al., Phys. Rev. Lett. 75, 1715 (1995).

[20] W. R. Phillips, I. Ahmad, D. W. Banes et al., Phys. Rev. Lett. 62, 1025 (1989).

[21] W. R. Phillips, J. Copnell, D. W. Banes et al., Phys. Rev. A 47, 3682 (1993).

[22] A. Akber, M. W. Reed, P. M. Walker et al., Phys. Rev. C 91, 031301 (2015).

[23] E. M. Burbidge, G. R. Burbidge, W. A. Fowler et al., Rev. Mod. Phys. 29, 547 (1957).

[24] Yu.N. Novikov, H. Schatz, P. Dendooven et al., Eur. Phys. J. A 11, 257 (2001).

[25] B. A. Watson, T. T. Bardin, J. A. Becker et al., Phys. Rev. Lett. 35, 1333 (1975).

[26] J. Schirmer, D. Habs, R. Kroth et al., Phys. Rev. Lett. 53, 1897 (1984).

[27] J. Kramp, D. Habs, R. Kroth et al., Nucl. Phys. A474, 412 (1987).

[28] C. Walz, H. Scheit, N. Pietralla et al., Nature 526, 406 (2015).

[29] M. Huyse, A. N. Andreyev, P. Van Duppen et al., Nature 405, 430 (2000).

[30] P. Dendooven, P. Decrock, M. Huyse et al., Phys. Lett. B 226, 27 (1989).

[31] D. R. Plante, W. R. Johnson and J. Sapirstein, Phys. Rev. A 49, 3519 (1994).

[32] W. Johnson and G. Soff, Atom. Data Nucl. Data 33, 405 (1985).

[33] Y. Litvinov, H. Geissel, Y. Novikov et al., Nucl. Phys. A734, 473 (2004).

[34] Y. Litvinov, H. Geissel, T. Radon et al., Nucl. Phys. A756, 3 (2005). 
[35] L. Chen, Y. A. Litvinov, W. R. Plaß et al., Phys. Rev. Lett. 102, 122503 (2009).

[36] M. W. Reed, I. J. Cullen, P. M. Walker et al., Phys. Rev. Lett. 105, 172501 (2010).

[37] M. W. Reed, P. M. Walker, I. J. Cullen et al., Phys. Rev. C 86, 054321 (2012).

[38] M. W. Reed, P. M. Walker, I. J. Cullen et al., J. Phys. Conf. Ser. 381, 2058 (2012).

[39] L. Chen, P. M. Walker, H. Geissel et al., Phys. Rev. Lett. 110, 122502 (2013).

[40] F. Bosch, H. Geissel, Y. Litvinov et al., Int. J. Mass. Spectrom. 251, 212 (2006).

[41] L. Chen, W. R. Plass, H. Geissel et al., Phys. Lett. B 691, 234 (2010).

[42] F. Nolden, P. Hülsmann, Y. Litvinov et al., Nucl. Instrum. Meth. A 659, 69 (2011).

[43] M. S. Sanjari, P. Hüllsmann, F. Nolden et al., Phys. Scripta T156, 014088 (2013).

[44] M. S. Sanjari, X. Chen, P. Hülsmann et al., Phys. Scripta T166, 014060 (2015).

[45] B. Franzke, H. Geissel and G. Münzenberg, Mass Spectrom. Rev. 27, 428 (2008).

[46] Y. Litvinov, F. Bosch, N. Winckler et al., Phys. Lett. B 664, 162 (2008).

[47] P. Kienle, F. Bosch, P. Bühler et al., Phys. Lett. B 726, 638 (2013).

[48] M. Steck, P. Beller, K. Beckert et al., Nucl. Instrum. Meth. A 532, 357 (2004).

[49] F. Nolden, K. Beckert, P. Beller et al., Nucl. Instrum. Meth. A 532, 329 (2004).

[50] H. Geissel, Y. Litvinov, F. Attallah et al., Nucl. Phys. A746, 150c (2004).

[51] M. Hausmann, J. Stadlmann, F. Attallah et al., Hyperfine Interact. 132, 289 (2001).
[52] H. Geissel, Y. A. Litvinov, K. Beckert et al., Eur. Phys. J.-Spec. Top. 150, 109 (2007).

[53] H. Geissel, P. Armbruster, K. Behr et al., Nucl. Instrum. Meth. B 70, 286 (1992).

[54] B. Franzke, Nucl. Instrum. Meth. B 24/25, 18 (1987).

[55] A. Musumarra, F. Farinon, C. Nociforo et al., AIP Conf. Proc. 1165, 415 (2009).

[56] C. Nociforo, F. Farinon, A. Musumarra et al., Phys. Scripta T150, 014028 (2012).

[57] F. Herfurth, Z. Andelkovic, W. Barth et al., Phys. Scripta T166, 014065 (2015).

[58] P. M. Hillenbrand, S. Hagmann, A. B. Voitkiv et al., Phys. Rev. A 90, 042713 (2014).

[59] P. M. Hillenbrand, S. Hagmann, D. H. JakubassaAmundsen et al., Phys. Rev. A 91, 022705 (2015).

[60] P. M. Hillenbrand, S. Hagmann, K. E. Stiebing et al., Phys. Scripta T166, 014026 (2015).

[61] FAIR, FAIR Baseline Technical Report 1, GSI, Darmstadt (2006)., http://www . fair-center .de/fileadmin/fair/ publications_FAIR/FAIR_BTR_1.pdf

[62] H. Geissel, H. Weick, M. Winkler et al., Nucl. Instrum. Meth. B 204, 71 (2003).

[63] A. Dolinskii, D. Berkaev, U. Blell et al., Phys. Scripta T166, 014040 (2015).

[64] T. Stöhlker, Y. A. Litvinov and for the SPARC Collaboration, Phys. Scripta T166, 014025 (2015).

[65] T. Stöhlker, V. Bagnoud, K. Blaum et al., Nucl. Instrum. Meth. B 365, 680 (2015).

[66] J. Yang, J. Xia, G. Xiao et al., Nucl. Instrum. Meth. B 317, 263 (2013).

[67] A. Ozawa, T. Uesaka, M. Wakasugi et al., Prog. Theor. Exp. Phys. 2012, $03 \mathrm{C009}$ (2012).

[68] T. Yamaguchi, Y. Yamaguchi and A. Ozawa, Int. J. Mass. Spectrom. 349-350, 240 (2013).

[69] Y. Litvinov, S. Bishop, K. Blaum et al., Nucl. Instrum. Meth. B 317, 603 (2013). 